\title{
El proceso de ruptura de la fuente del terremoto de Ecuador 2016 (7.8 Mw)
}

\author{
César Jiménez ${ }^{*}{ }^{1}$, Miguel Saavedra J. ${ }^{1}$, Daniel Olcese ${ }^{2}$ y Nick Moreno ${ }^{1}$ \\ ${ }^{1}$ Universidad Nacional Mayor de San Marcos, Lima, Perú \\ ${ }^{2}$ Universidad Nacional de Ingeniería, Lima, Perú
}

Recibido 03 diciembre 2019 - Aceptado 15 enero 2020

\begin{abstract}
Resumen
El terremoto de Ecuador de 2016 (7.8 Mw), forma parte de la secuencia de grandes sismos en Ecuador y Colombia: 1906 (8.4 a 8.8 Mw), 1942 (7.8 Mw), 1958 (7.7 Mw), 1979 (8.2 Mw), todos con un mecanismo focal del tipo de falla inversa. En esta investigación se ha obtenido la distribución de la fuente sísmica (que indica la presencia de 2 asperezas principales) a partir de inversión de señales telesísmicas, así como los parámetros del proceso de ruptura sísmica: mecanismo focal que indica un tipo de falla inversa $\left(\right.$ strike $=25^{\circ}, \operatorname{dip}=12^{\circ}$, rake $=118^{\circ}$ ) para una profundidad focal de $23 \mathrm{~km}$ y la función temporal de la fuente sísmica que indica un evento compuesto con una duración del proceso de ruptura de alrededor de $70 \mathrm{~s}$, asumiendo una velocidad de ruptura promedio de $3.0 \mathrm{~km} / \mathrm{s}$. El momento sísmico calculado fue de $5.74 \times 10^{20} \mathrm{Nm}$, lo que equivale a una magnitud de $7.8 \mathrm{Mw}$. La aspereza más pequeña se localiza alrededor del epicentro y la aspereza principal se ubica al sur-este del epicentro en mar y en continente con una dislocación (slip) máxima de $3.8 \mathrm{~m}$. En este caso, el patrón de deformación cosísmica vertical no favorece la generación de un tsunami grande.
\end{abstract}

Palabras clave: Terremoto de Ecuador, inversión sísmica, mecanismo focal.

\section{The rupture process of the source of the 2016 Ecuador earthquake (Mw 7.8)}

\begin{abstract}
The 2016 Ecuador earthquake ( $\mathrm{Mw} 7.8$ ), is part of the sequence of large earthquakes in Ecuador and Colombia: 1906 (8.4 to $8.8 \mathrm{Mw}$ ), 1942 (Mw 7.8), 1958 ( Mw 7.7), 1979 ( $\mathrm{Mw} 8.2$ ), all these events with a focal mechanism of the thrust fault type. In this research, we have obtain the seismic source distribution (indicating the presence of 2 main asperities), from inversion of teleseismic signals, as well as the parameters of the seismic rupture process: focal mechanism that indicates a type of inverse fault $\left(\right.$ strike $=25^{\circ}$, dip $=12^{\circ}$, rake $=118^{\circ}$ ) for a focal depth of $23 \mathrm{~km}$ and the source time function indicating a composite event, the duration of the rupture process was around $70 \mathrm{~s}$, assuming an average rupture velocity of $3.0 \mathrm{~km} / \mathrm{s}$. The seismic moment was calculated at $5.74 \times 10^{20} \mathrm{Nm}$, equivalent to a magnitude of $\mathrm{Mw}$ 7.8. The smallest asperity is located around the epicenter and the main asperity is located around the coastline to the south-east of the epicenter. The maximum dislocation or slip was $3.8 \mathrm{~m}$. In this case, the vertical coseismic deformation pattern does not favor the generation of a large tsunami.
\end{abstract}

Keywords: Ecuador earthquake, seismic inversion, focal mechanism.

\section{Introducción}

En general, una señal geofísica (conjunto de datos o serie de tiempo) contiene información del proceso físico generador del fenómeno, del medio sobre el cual se ha propagado la perturbación (ondas) y del instrumento sobre el cual se ha registrado la señal. Si se conocen y se remueven los efectos de las propiedades del medio y de los parámetros del instrumento, es posible determinar los parámetros del proceso generador del fenómeno (en este caso, la fuente sísmica) a partir de las señales o datos observados (ondas sísmicas).

Las señales sísmicas contienen información sobre la fuente sísmica, pero también dependen de la estructura de la Tierra a traveś de la cual se propagan las ondas

\footnotetext{
*cjimenezt@unmsm.edu.pe
} 
sísmicas y del instrumento sobre el cual son registradas. Para las ondas sísmicas, el efecto más importante sobre la propagación es la estructura de velocidades de la Tierra. Una vez que se ha tomado en cuenta los efectos de propagación y del instrumento, las formas de ondas sísmicas pueder ser utilizadas para determinar los parámetros de la fuente sísmica mediante un proceso de inversión (Johnson, 1999) [Joh99].

Las formas de onda telesísmicas de volumen pueden resolver variaciones temporales y espaciales del proceso de ruptura sísmica, a lo largo de la dirección del azimut. Sin embargo, la resolución en la dirección de la profundidad, o a lo largo del buzamiento, es relativamente pobre (Satake, 1993).

El 16 de abril de 2016 a las 18:58 (hora local) ocurrió un fuerte sismo en el litoral norte de Ecuador, con epicentro localizado a $37 \mathrm{~km}$ al noreste de la ciudad de Pedernales, la cual fue una de las más afectadas. El número de víctimas mortales alcanzó alrededor de 670 en toda la nación. Este evento sísmico fue sentido hasta Cali-Colombia por el norte y Chiclayo-Perú por el sur.

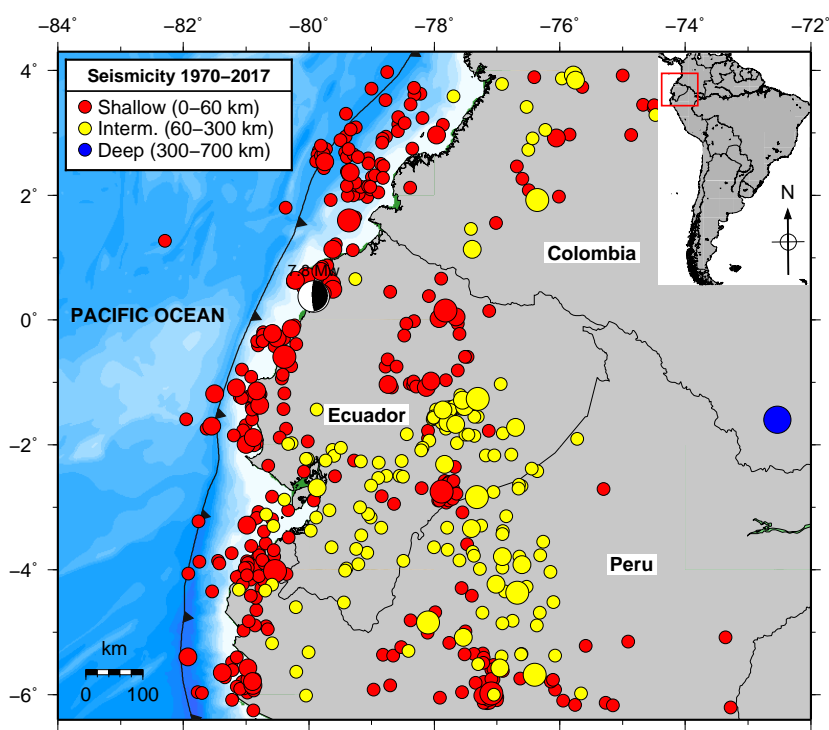

Figura 1: Mapa de la sismicidad de Ecuador. La fuente del terremoto de Ecuador 2016 está representada por el diagrama focal.

\section{Sismicidad y sismotectónica de Ecuador}

La zona litoral del Ecuador (y Colombia) es parte del Cinturón Sísmico del Pacífico, por lo que es escenario de ocurrencia de grandes sismos y tsunamis a lo largo de su historia, debido al proceso de subducción oblicua entre la placa de Nazca y la placa Continental Sudamericana con una velocidad de convergencia relativa de 55 mm/año (Kendrick et al., 2003) [Ken03]. Como una consecuencia de la subducción oblicua, el área de los Andes nor-oeste se ha separado de la placa estable de Sudamérica y se mueve en dirección nor-este (Nocquet et al., 2014). La Figura 1 muestra la distribución de la sismicidad de Ecuador, con presencia de sismos superficiales ubicados principalmente entre la costa y la fosa marina, sismos intermedios en la región de los Andes y sismos de foco profundo en la selva colombiana (como el de 1970 de magnitud $8.1 \mathrm{Mw}$ ). La presencia de volcanismo activo en los Andes de Ecuador se debe al tipo de subducción normal en esta región, similar al tipo de subducción del sur del Perú.

El año de 1906 ocurrió un gran terremoto en la región norte de Ecuador y sur de Colombia (Figura 2), con una magnitud estimada en $8.8 \mathrm{Mw}$ (Kanamori y McNally, 1982) [Kan82]. Okal (1992) estimó una magnitud de 8.5$8.6 \mathrm{M}_{m}$, basada en el cálculo de ondas sísmicas superficiales [Okal92]. Sin embargo, investigaciones recientes han estimado la magnitud de este evento en $8.4 \mathrm{Mw}$ (Yoshimoto et al., 2017) [Yos17] y 8.3 a $8.6 \mathrm{Mw}$ (Yamanaka et al., 2017) [Yam17] a partir de inversión de ondas de maremoto.

Como parte de la secuencia sísmica de EcuadorColombia posterior a 1906, se produjeron los eventos sísmicos de 1942 (7.8 Mw), 1958 (7.7 Mw), 1979 (8.2 Mw) (Herd et al., 1981) [Her81] y recientemente el de 2016 (7.8 $\mathrm{Mw})$ que es probablemente una repetición del evento de 1942, por tener la misma magnitud y la misma región de ruptura (Ye et al., 2016) [Yet16]. Tanto el evento sísmico de 1906 como el de 1979 generaron devastadores tsunamis (Soloviev, 1975; Herd et al., 1981).

El evento sísmico de Colombia-Ecuador de 1906 tuvo un área de ruptura de alrededor de $500 \mathrm{~km} \mathrm{y} \mathrm{un} \mathrm{pro-}$ ceso de ruptura múltiple o compuesto (Kelleher, 1972) [Kel72]. Sin embargo, el modo de ruptura sísmica ha cambiado a partir de la secuencia sísmica que inició en 1942, donde cada evento sísmico (1942, 1958, 1979 y 2016) ha fracturado una pequeña porción del área mayor, con un proceso de ruptura simple (Swenson y Beck, 1996) [Swe96].

En la Figura 2 se observa que en la parte norte del área de ruptura del evento de 1906 (correspondiente al litoral colombiano), no ha habido grandes terremotos en el siglo XX y en lo que va del siglo XXI, por lo que esta zona tendría un alto potencial para la generación de sismos tsunamigénicos, espcecialmente al norte de la latitud $2.5^{\circ}$, que sería el límite de la región de ruptura del terremoto de 1979 (8.2 Mw).

\section{Antecedentes de estudios previos}

Varios investigadores han estudiado al evento sísmico de Ecuador 2016, en sus aspectos sismológicos, tsunamigénicos y geodésicos, entre otros se mencionan a los siguientes: 


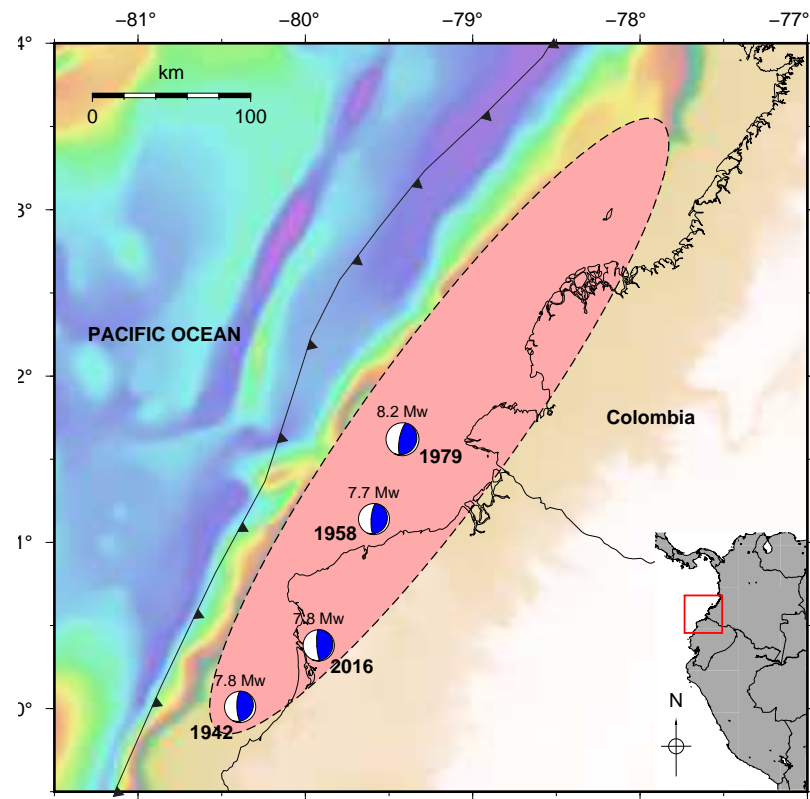

Figura 2: El ovalo rosado representa el área de ruptura del terremoto de Ecuador-Colombia de 1906. La secuencia sísmica 1942-1958-1979-2016 está representada por los diagramas focales.

Ye et al. (2016) utilizaron datos telesísmicos para obtener la distribución de asperezas del terremoto de Ecuador 2016, a partir de este modelo de fuente simularon la propagación del tsunami en 4 estaciones mareográficas. Proponen que el evento del 2016 es una repetición del terremoto de 1942. [Yet16].

Nocquet et al. (2016), combinaron resultados de sismicidad histórica, datos geodésicos y observaciones locales del terremoto de Ecuador (7.8 Mw) para reconstruir el estado de esfuerzos en esta zona de subducción desde el gran terremoto de 1906. Demostraron que el terremoto de Pedernales de 2016 implicó la ruptura sucesiva de las dos asperezas en la interfaz de la placa que estaban bloqueados antes del terremoto [Noc16].

Yoshimoto et al. (2017) realizaron la inversión de datos telesísmicos del evento de Ecuador 2016, también llevaron a cabo una inversión de formas de onda del tsunami de Ecuador-Colombia de 1906 y estimaron la magnitud de este evento histórico en 8.4 Mw [Yos17].

Heidarzadeh et al. (2017), utilizaron registros telesísmicos para obtener mediante inversión varios modelos de fuente sísmica, los cuales fueron verificados mediante simulación del tsunami correspondiente para obtener mareogramas sintéticos que fueron comparados con los observados, con lo cual se obtuvo un modelo de fuente sísmica robusto que cumplía con las condiciones del tsunami [Hei17].

He et al. (2017) utizaron imágenes satelitales InSAR (Interferometría de Radar de Apertura Sintética), para determinar los desplazamientos cosísmicos en la superficie asociados con el terremoto de Ecuador 2016. La máxima dislocación (slip) inferida es de $2.5 \mathrm{~m}$ [Het17].

Yi et al. (2018) realizaron la inversión combinada de señales telesísmicas y datos InSAR para obtener la distribución de la fuente sísmica. La velocidad de ruptura óptima se estimó en $2.0 \mathrm{~km} / \mathrm{s}$, el momento sísmico se estimó en $5.75 \times 10^{20} \mathrm{Nm}(7.8 \mathrm{Mw})$. Sugieren que probablemente las áreas de ruptura de los eventos de 1942 y 2016 no se solapan [Yit18].

Mothes et al. (2018), mediante datos de la red geodésica del Ecuador, obtuvieron los desplazamientos cosísmicos debido al terremoto de Ecuador 2016, con desplazamiento horizontales de hasta $80 \mathrm{~cm}$ y desplazamientos transitorios de hasta $2 \mathrm{~m}$ pico a pico, en el campo cercano. El patrón de acoplamiento intersísmico sugiere que el segmento Esmeraldas-Nariño tiene un potencial sísmico particularmente alto [Mot18].

En esta investigación se ha utilizado las señales telesísmicas para caracterizar el proceso de ruptura sísmica y obtener el mecanismo focal así como la distribución de la fuente sísmica a través de un proceso de inversión de formas de ondas telesísmicas mediante el modelo numérico de Kikuchi y Kanamori (1991) [Kik91]. Adicionalmente, se ha calculado el patrón de deformación cosísmica.

\begin{tabular}{ll}
\hline Fecha & 16 Abril 2016 \\
\hline Tiempo origen UTC & $23: 58: 36$ \\
Latitud & $00.3819^{\circ}$ \\
Longitud & $-79.9218^{\circ}$ \\
Profundidad & $20 \mathrm{~km}$ \\
Magnitud & $7.8 \mathrm{Mw}$ \\
Strike & $\theta=25^{\circ}$ \\
Dip & $\delta=12^{\circ}$ \\
Rake & $\lambda=124^{\circ}$ \\
\hline
\end{tabular}

Tabla 1: Los parametros hipocentrales del terremoto de Ecuador 2016 fueron reportados por el National Earthquake Information Center (NEIC). El mecanismo focal fue reportado por el Global Centroid Moment Tensor (CMT).

\section{Adquisición y procesamiento de datos}

Los parámetros hipocentrales del NEIC (https: //earthquake.usgs.gov/earthquakes) y el mecanismo focal del CMT (www.globalcmt.org) del terremoto de Ecuador 2016 se muestran en la Tabla 1. Los datos de topografía global se han tomado del modelo Gebco (https://www.gebco.net) con una resolución de 30 segundos de arco. Las señales sísmicas se adquirieron de 63 estaciones telesísmicas de la red mundial Incorporated Research Institutions for Seismology (IRIS) (http://ds.iris.edu/wilber3/) para una distancia epicentral entre $30^{\circ}$ y $90^{\circ}$. En general, se observa una buena 
distribución azimutal de las estaciones, nótese que alrededor del azimut de $270^{\circ}$ (en el Océano Pacífico) hay pocas estaciones y la mayor concentración de estaciones está en el hemisferio norte (Figura 3).

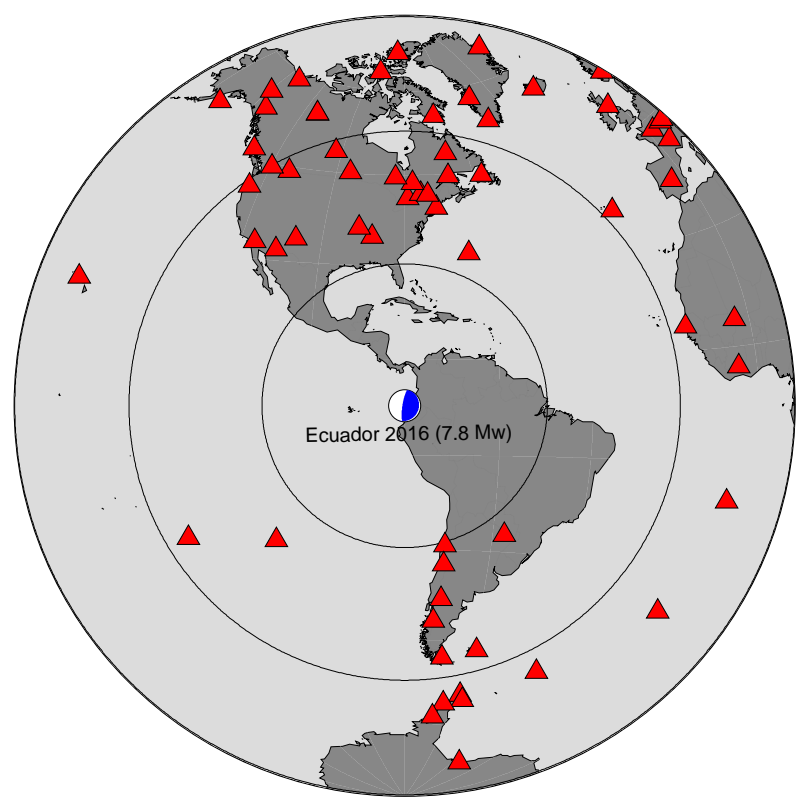

Figura 3: Ubicación de las 63 estaciones telesísmicas (triángulo rojo) utilizadas en el proceso de inversión, la fuente está representada por el diagrama focal y los círculos concéntricos representan distancias epicentrales de $30^{\circ}, 60^{\circ}$ y $90^{\circ}$.

Sólo se ha escogido a las señales sísmicas cuya relación señal-ruido es mayor a 10, para lo cual se ha utilizado el software GrafSac (Jiménez, 2007). Se ha tomado una ventana de tiempo de la señal de $20 \mathrm{~s}$ antes del inicio de la onda P y 120 s después del mismo. Se realizó la deconvolución de la señal para remover la respuesta del instrumento a través del software Seismic Analysis Code (SAC: https://ds.iris.edu/files/sac-manual).

Luego, se realizó la integración de la señal para obtener el registro en desplazamiento con un intervalo de muestreo de $0.5 \mathrm{~s}$. Se aplicó un filtro pasa banda entre las frecuencias de corte de $0.01 \mathrm{~Hz}$ y $0.10 \mathrm{~Hz}$, correspondiente a los periodos en el rango de $10 \mathrm{~s}$ y $100 \mathrm{~s}$. Las señales sísmicas fueron rotadas en sus componentes radial y tangencial para obtener las ondas $\mathrm{P}$ y SH. Se aplicó filtros de atenuación con una constante de tiempo de 1 s y $4 \mathrm{~s}$ para las ondas $\mathrm{P}$ y $\mathrm{SH}$ respectivamente.

Para obtener las funciones de Green o sismogramas elementales, se utilizó un modelo de velocidades global, a la cual se añadió una capa adicional de $3 \mathrm{~km}$ de espesor para modelar el comportamiento de las ondas $\mathrm{P}$ en el océano en la vecindad de la fuente (Tabla 2).

\section{Inversión de formas de onda telesísmicas}

Para calcular el mecanismo focal y la distribución de la fuente sísmica, se ha utilizado el método de inversión de formas de ondas telesísmicas de Kikuchi y Kanamori (2003) [Kik03].

La física del proceso directo y del proceso inverso se explica de la siguiente manera: en el primer caso, a partir de los parámetros de la fuente y de las ecuaciones de propagación de ondas sísmicas, es posible obtener los sismogramas sintéticos en una ubicación determinada. Mientras que en el segundo caso, a partir de los datos observados en uno o varios puntos de observación y teniendo en cuenta las ecuaciones que gobiernan al sistema (propagación de ondas sísmicas), se estiman los parámetros del modelo que caracteriza a la fuente sísmica.

En los problemas inversos hay una relación matemática que enlaza los datos observados (que se llamará el vector de datos) con los parámetros del modelo que se desea conocer (que se llamará el modelo), en este caso, los valores de la dislocación (slip) para cada subfuente.

Los valores del mecanismo focal pueden ser tomados del catálogo del Global CMT (página web: www. globalcmt.org); luego, estos valores se afinan mediante el proceso de inversión de ondas telesísmicas. Las dimensiones del área de ruptura de los grandes sismos pueden determinarse a partir de la distribución de las réplicas dentro de los primeros días (Kanamori, 1977) [Kan77], [Kan82]. Esto deja sólo un parámetro a ser calculado: la dislocación o slip. El valor de la dislocación es una medida del tamaño de la aspereza respectiva y está asociada al momento sísmico escalar, el cual es una medida del tamaño del terremoto.

\begin{tabular}{lcccr}
\hline$N$ & $v_{p}(\mathrm{~km} / \mathrm{s})$ & $v_{s}(\mathrm{~km} / \mathrm{s})$ & $\rho\left(\mathrm{g} / \mathrm{cm}^{3}\right)$ & $t(\mathrm{~km})$ \\
\hline 1 & 1.50 & 0.00 & 1.00 & 3.0 \\
2 & 6.50 & 3.74 & 2.87 & 15.0 \\
3 & 7.80 & 4.40 & 3.30 & $\infty$ \\
\hline
\end{tabular}

Tabla 2: Modelo de estructura de velocidad de ondas sísmicas en la vecindad de la fuente. El parámetro $t$ representa el espesor de la capa litosférica.

Para los sismos de gran magnitud, la distribución de la dislocación sobre el área de ruptura no es homogénea, sino que está constituida en forma heterogénea por varias asperezas o zonas de mayor liberación de energía sísmica. El método de inversión sísmica puede determinar la distribución de dichas asperezas sobre el plano de ruptura. 

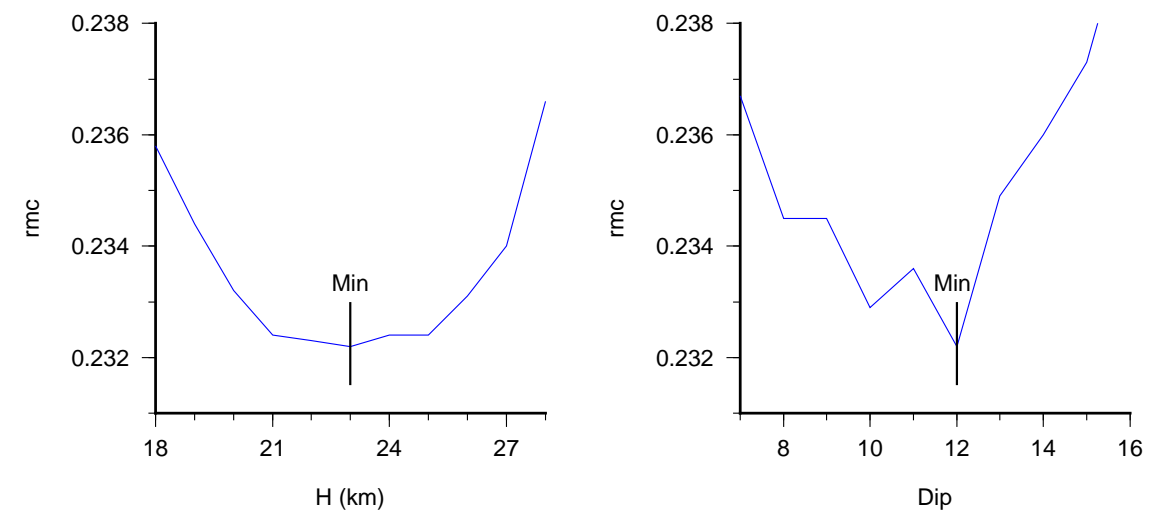

Figura 4: Diagramas de la prueba de sensibilidad para obtener la profundidad $(H=23 \mathrm{~km})$ y ángulo de buzamiento $\left(\right.$ dip $\left.=12^{\circ}\right)$ óptimos, que corresponden al menor residual o error.

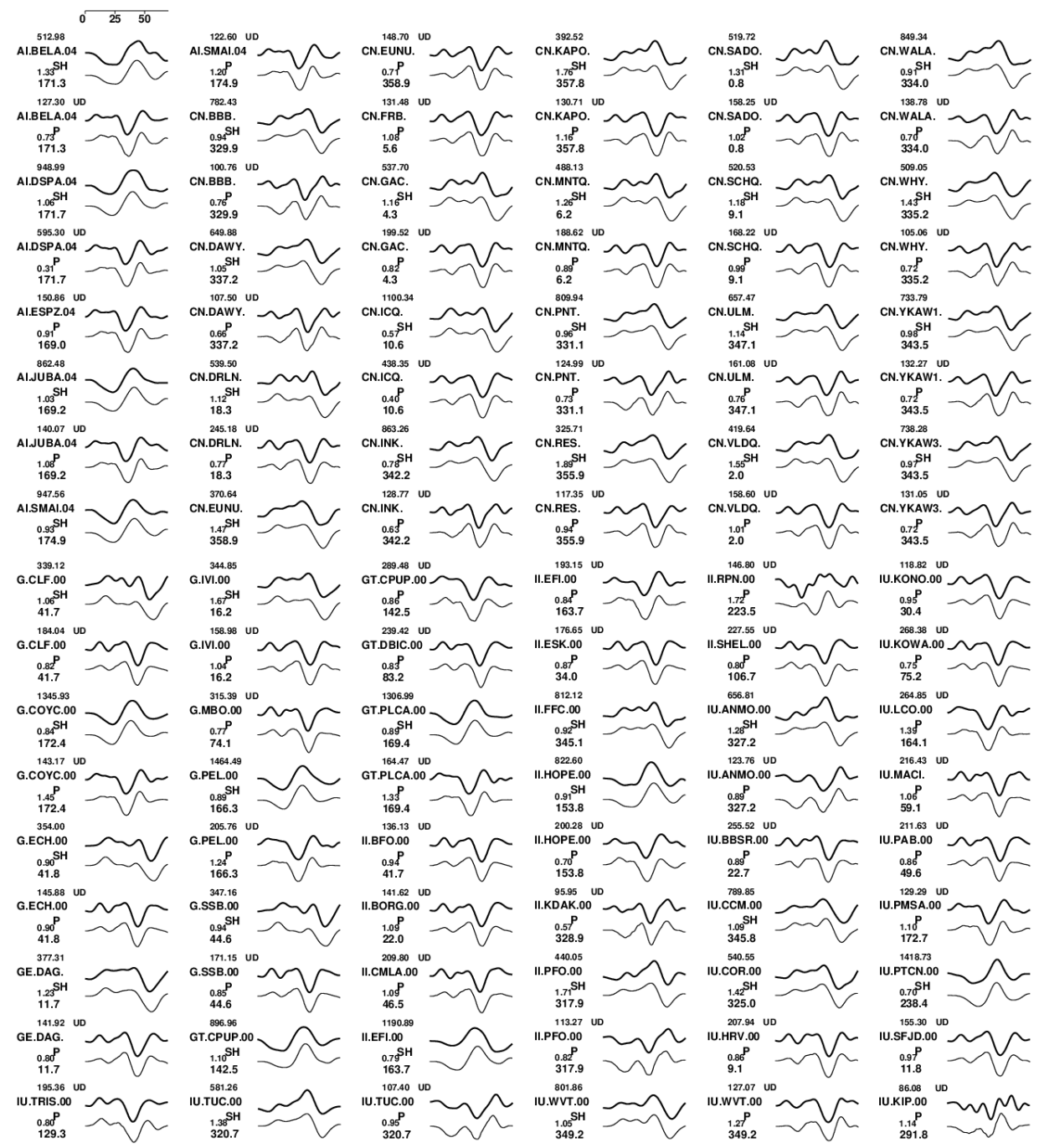

Figura 5: Comparación de sismogramas observados y simulados del terremoto de Ecuador 2016. La señal observada en la parte superior y la señal sintética en la parte inferior. 
La geometría de ruptura del terremoto es dividida en varias subfuentes rectangulares más pequeñas, en este caso $7 \times 11$ (Figura 7 ). Las dimensiones de cada subfuente $(15 \mathrm{~km} \times 15 \mathrm{~km})$ así como el ángulo de buzamiento $\left(\operatorname{dip}=12^{\circ}\right)$ y azimut $\left(\right.$ strike $\left.=25^{\circ}\right)$ son iguales para cada subfuente. La amplitud de la dislocación (slip) y el ángulo de dislocación son calculados mediante el proceso de inversión para cada subfuente.

Para obtener la función temporal de la fuente sísmica se utilizaron 6 funciones triangulares de ancho 6 s separados por $4 \mathrm{~s}$, cuyas amplitudes fueron calculadas durante el proceso de inversión.

Se realizó una prueba de sensibilidad para afinar los resultados de la profundidad focal y el ángulo de buzamiento, para los que se obtuvieron los valores de $23 \mathrm{~km}$ y $12^{\circ}$ respectivamente correspondientes al mínimo residual de 0.2322 (Figura 4).

\section{Resultados y Discusión}

En esta sección se presentan los resultados obtenidos en esta investigación, los cuales son muy similares a los obtenidos por otros investigadores (Ye et al., 2016; Nocquet et al., 2016; Heidarzadeh et al., 2017). Por ejemplo, todos consideran que hubieron 2 asperezas y que la mayor aspereza está ubicada en Continente, así también este evento sísmico es una repetición del evento de 1942 ( Mw 7.8).

Los sismogramas observados y calculados (sintéticos) mediante el proceso de inversión, se muestran en la Figura 5. La mayoría de los pares de señales sísmicas tienen una buena correlación, con excepción de las señales de la estación sísmica de KIP, situada en la isla de Hawaii, donde por lo general, las señales son ruidosas. El error o varianza normalizada total fue de $0.23 \mathrm{~s}$.

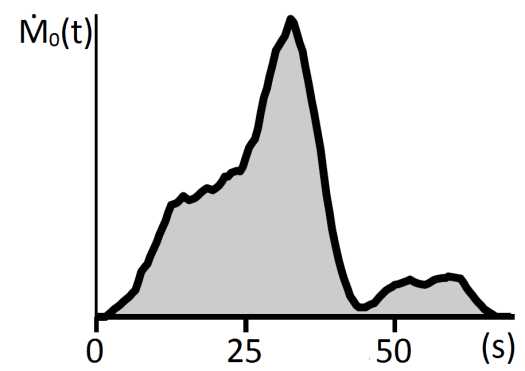

Figura 6: Función temporal de la fuente sísmica del terremoto de Ecuador 2016. La duración del proceso de ruptura fue de alrededor de $70 \mathrm{~s}$.

La función temporal de la fuente sísmica refleja el carácter de la complejidad de la fuente sísmica (Figura 6). Se puede observar hasta 3 momentos de liberación de energía sísmica. El primero corresponde desde el inicio hasta los $20 \mathrm{~s}$. La máxima liberación de energía sísmica corresponde al intervalo entre los 20 y 45 s. Finalmente, en el tramo de 45 a 70 s hay una ligera liberación de energía sísmica. Se ha asumido una velocidad de ruptura promedio $3 \mathrm{~km} / \mathrm{s}$.

La Figura 7 muestra la distribución de la fuente sísmica, nótese que el área de ruptura del terremoto de Ecuador 2016 abarca parte del océano y parte de continente.Es por esto que el tsunami generado fue relativamente pequeño, lo que implica que no es posible recuperar la fuente sísmica utilizando solo inversión de ondas de maremoto, por cuanto las asperezas ubicadas en continente no generan tsunami.

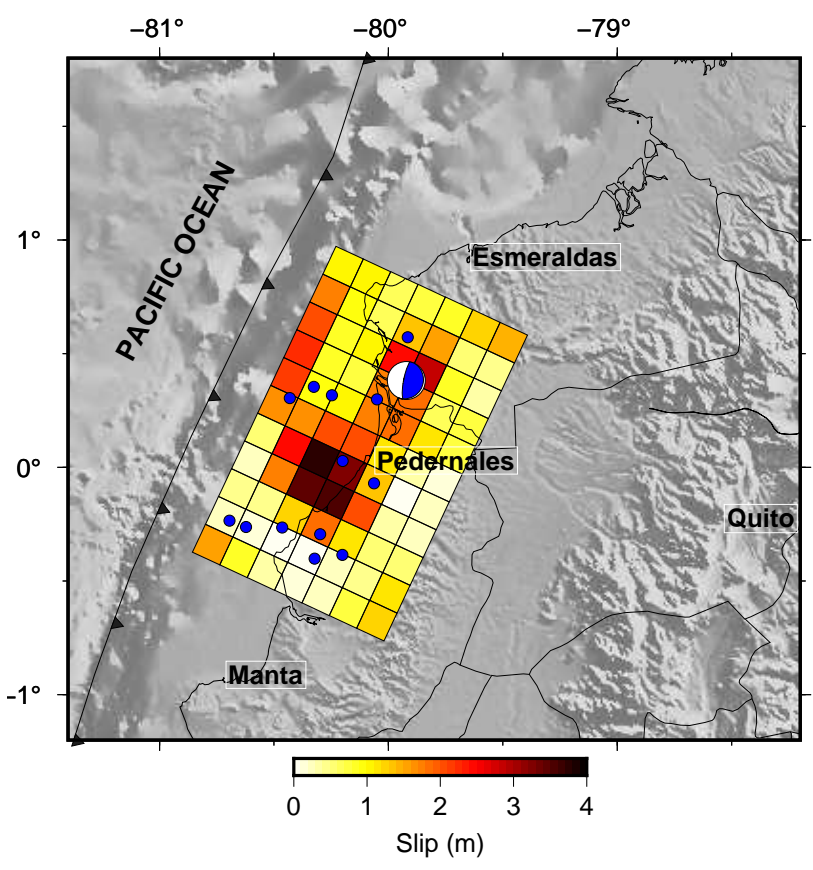

Figura 7: Distribución de la fuente sísmica del terremoto de Ecuador 2016. Nótese que las asperezas principales están ubicadas en continente y en el mar. Cada subfuente tiene una dimensión de $15 \mathrm{~km} \times 15 \mathrm{~km}$. Los pequeños círculos azules representan a las réplicas en el primer día.

La Figura 8 muestra un mapa con la deformación cosísmica vertical, la cual se obtuvo a partir de la formulación de Okada (1992) [Oka92]. El máximo levantamiento fue de $0.82 \mathrm{~m}$ y la máxima subsidencia fue de $0.29 \mathrm{~m}$.

El levantamiento cosísmico ocurrió en mar y continente, esto explica la generación de un pequeño tsunami local sin mayor impacto, a diferencia de otros tsunamis de igual o menor magnitud pero con un mayor impacto, como el de Lambayeque-Perú de 1960 (7.6 Mw) y el de Chimbote-Perú de 1996 (7.5 Mw) que ocasionó 12 víctimas mortales. 


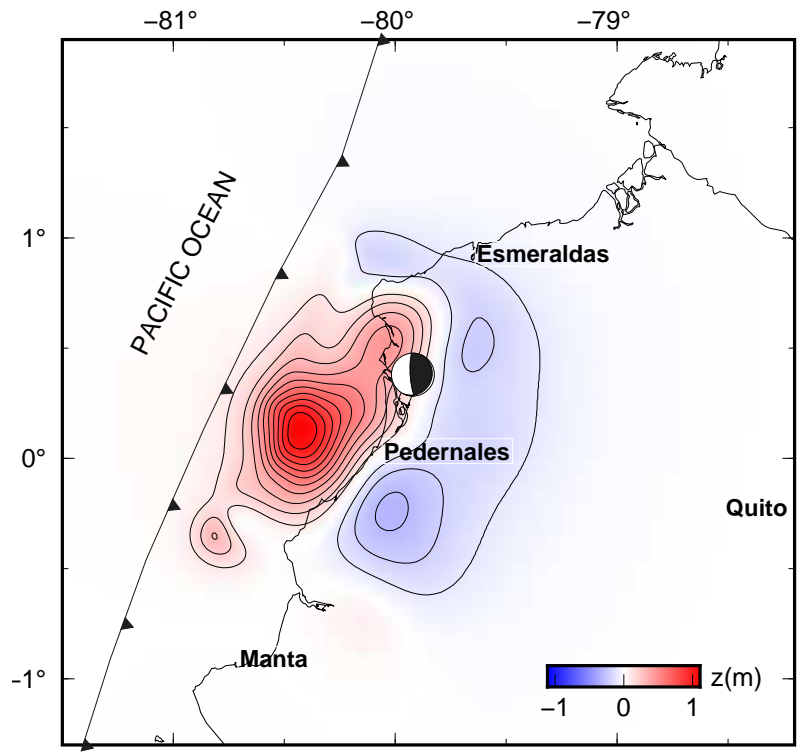

Figura 8: Deformación vertical debido al terremoto de Ecuador 2016. Nótese que la mayor parte del levantamiento cosísmico (en color rojo) ocurrió en el mar. Cada curva de nivel representa $10 \mathrm{~cm}$ de elevación o subsidencia.

\section{Conclusiones}

Los parámetros del plano de ruptura o mecanismo focal obtenido indican un tipo de falla inversa cuyos parámetros son: strike $=25^{\circ}, \operatorname{dip}=12^{\circ}$, rake $=118^{\circ}$; para una profundidad focal estimada en $23 \mathrm{~km}$, coherente con el patrón sismotectónico de la región de Ecuador-Colombia.

La función temporal de la fuente sísmica indica un evento compuesto por 2 secuencias principales de liberación de energía sísmica (correspondiente a 2 asperezas principales), con una duración total del proceso de ruptura de alrededor de $70 \mathrm{~s}$, para una velocidad de ruptura promedio de $3.0 \mathrm{~km} / \mathrm{s}$.

La máxima dislocación (slip) fue de $3.8 \mathrm{~m}$, que corresponde a la aspereza principal ubicada al sur del epicentro (en el Continente) y muy cerca de la ciudad de Pedernales, la que sufrió el mayor impacto sobre la infraestrutura y la mayor cantidad de víctimas.

Las dimensiones del plano de ruptura se han estimado en $165 \mathrm{~km} \times 105 \mathrm{~km}$, coherente con la distribución de las réplicas. El tamaño del terremoto es cuantificado por su momento sísmico, cuyo valor obtenido mediante el proceso de inversión fue de $5.74 \times 10^{20} \mathrm{Nm}$, equivalente a una magnitud de $7.8 \mathrm{Mw}$.

El patrón de deformación cosísmica vertical no favorece la generación de un tsunami grande, debido a la pequeña amplitud de deformación vertical (alrededor de $1 \mathrm{~m})$ y debido a que una parte del levantamiento cosísmico está ubicado en tierra. Sin embargo, las estaciones mareográficas del Ecuador registraron perturbaciones de pequeña amplitud menor a medio metro.

Teniendo en cuenta las similitudes de mecanismo focal, magnitud, distribución de réplicas y geometría de la fuente sísmica de los eventos de 1942 y 2016, se infiere que este último es una repetición del primero. Sin embargo, aún existe un alto potencial de generación de sismos de gran magnitud $(\mathrm{Mw}>7.0)$ en esta región entre la línea de costa y la fosa marina.

\section{Referencias}

[Het17] He, P., Hetland, E., Wang, Q., Ding, K., Wen, Y., and Zou, R. (2017). Coseismic slip in the 2016 Mw7.8 Ecuador earthquake imaged from Sentinel1A Radar Interferometry. SRL, vol 88, No. 2A, pp $1-10$.

[Hei17] Heidarzadeh, M., Murotani, S., Satake, K., Takagawa, T. and Saito, T. (2017). Fault size and depth extent of the Ecuador earthquake (Mw 7.8) of 16 April 2016 from teleseismic and tsunami data. GRL, vol 44, pp 2211-2219.

[Her81] Herd, D., Youd, T., Meyerr, H., Arango, J., Person, W. and Mendoza, C. (1981). The great Tumaco, Colombia earthquake of 12 December 1979. Science, vol 211, No. 4481, pp 441-445.

[Jim07] Jiménez, C. (2007). Procesamiento digital de se- ñales sísmicas con Matlab. Revista de Investigación de Física, vol 10, No. 2, pp 45-50.

[Joh99] Johnson, J. (1999). Heterogeneous coupling along Alaska-Aleutians as inferred from tsunami, seismic and geodetic inversions. Advances in Geophysics, vol 39, pp 1-116.

[Kan77] Kanamori, H. (1977). The energy release in great earthquakes. JGR, vol 82, pp 2981-2987.

[Kan82] Kanamori, H. y McNally, K. (1982). Variable rupture mode of the subduction zone along the Ecuador Colombia coast. BSSA, vol 72, No. 4, pp 1241-1253.

[Kel72] Kelleher, J. (1972). Rupture zones of large South American earthquakes and some predictions. JGR, vol 77, No. 11, pp 2087-2103.

[Ken03] Kendrick, E., Bevis, M., Smalley, J., Brooks, R., Vargas, R. Lauria, E., Souto, L. (2003). The Nazca 
South America Euler vector and its rate of change. J. South Am. Eart Sci., vol 16(2), pp 125-131.

[Kik91] Kikuchi, M. and Kanamori, H. (1991). Inversion of Complex Body Waves. Bulletin of Seismological Society of America, Vol. 81, No 6, pp 2335-2350.

[Kik03] Kikuchi, M. and Kanamori, H. (2003). Notes on Teleseismic Body-Wave Inversion Program: http: //www.eri.u-tokyo.ac.jp/ETAL/KIKUCHI

[Mot18] Mothes, P., Rolandone, F., Nocquet, J., Jarrin, P., Alvarado, A., Ruiz, M., Cisneros, D., Paez, H. and Segovia, M. (2018). Monitoring the earthquake cycle in the northern Andes from the Ecuadorian cGPS network. SRL, vol 89, No. 2A, pp 534-541.

[Noc14] Nocquet, J., Villegas, J., Chlieh, M., Mothes, P., Rolandone, F., Jarrín, P., Cisneros, D., Alvarado, A. Audin, L. Bondoux, F. (2014). Motion of continental slivers and creeping subduction in the northern Andes. Nature Geosciences, doi: 10.1038/NGEO2099.

[Noc16] Nocquet, J., Jarrin, P., Valle, M., Mothes, P., Grandin, R., Rolandone, F., Delouis, B., Yepes, H., Font, Y., Fuentes, D., Regnier, M., Cisneros, D., Sladen, A. (2014). Supercycle at the Ecuadorian subduction after the 2016 Pedernalesn earthquake. Nature Geoscience, doi: 10.1038/NGEO2864.

[Oka92] Okada, Y. Internal deformation due to shear and tensile faults in a half space. Bulletin of Seismological Society of America Vol. 82, No. 2, pp 1018-1040, (1992).

[Okal92] Okal, E. (1992). Use of the mantle magnitude $\mathrm{Mm}$ for the reassessment of the moment of historical earthquake. PAAG, vol 139, No. 1, pp 17-57.

[Sat93] Satake, K. (1993). Depth distribution of coseismic slip along the Nankai Trough, Japan, from joint inversion of geodetic and tsunami data. JGR, vol 98, No. B3, pp 4553-4565.

[Sol75] Soloviev, K. and Go, Ch. (1975). Catalogue of Tsunamis on the Eastern Shore of the Pacific Ocean (1513-1968). Nauka Publishing House, Moscow, pp 204.

[Swe96] Swenson, J. y Beck, S. (1996). Historical 1942 Ecuador and 1942 Peru subduction earthquakes, and earthquakes cycles along Colombia-Ecuador and Peru subduction segments. PAAG, vol 146, No. 1 , pp 67-101.

[Yam17] Yamanaka, Y., Tanioka, Y. and Shiina, T. (2017). A long source area of the 1906 ColombiaEcuador earthquake estimated from observed tsunami waveforms. Earth, Planets and Space, vol 69:163, pp 1-11.

[Yet16] Ye, L., Kanamori, H., Avouac J., Li, L., Fai, K. and Lay, T. (2016). The 16 April 2016, Mw 7.8 Ecuador earthquake: A quasi repeat of the 1942 Ms 7.5 earthquake and partial re-rupture of the 1906 Ms8.6 Colombia-Ecuador earthquake. Earth and Planetary Science Letters, vol 454, pp 248-258.

[Yit18] Yi, L., Xu, C., Wen, Y., Zhang, X. and Jiang, G. (2018). Rupture process of the 2016 Mw7.8 Ecuador earthquake from joint inversion of InSAR data and teleseismic P waveforms. Tectonophysics, vol 722, pp 163-174.

[Yos17] Yoshimoto, M., Kumagai, H., Acero, W., Ponce, G., Vásconez, F., Arrais, S., Ruiz, M., Alvarado, A., Pedraza, P., Dionicio, V., Chamorro, O., Maeda, Y. and Nakano, M. (2017). Depth-dependent rupture mode along Ecuador-Colombia subduction zone. GRL, vol 44, pp 2203-2210. 